\title{
Boundary value problems of the nonlinear multiple base points impulsive fractional differential equations with constant coefficients
}

Yunsong Miao and Fang Li*

"Correspondence:

fangli860@gmail.com School of Mathematics, Yunnan

Normal University, Kunming, 650092, P.R. China

\begin{abstract}
In this paper, the nonlinear multiple base points boundary value problems of the impulsive fractional differential equations are studied. By using the fixed point theorem and the Mittag-Leffler functions, the sufficient conditions for the existence of the solutions to the given problems are formulated. An example is presented to illustrate the result.
\end{abstract}

MSC: 34A08; 34A37; 34B10

Keywords: nonlinear multiple base points fractional differential equations; boundary value problem; impulsive condition; Mittag-Leffler functions

\section{Introduction}

The application of fractional calculus is very broad, including characterization of mechanics and electricity, earthquake analysis, the memory of many kinds of material, electronic circuits, electrolysis chemical, etc. ([1-5]). In recent years, there has been a significant development in solving differential equations involving fractional derivatives ([6-14] and the references therein).

In the left and right fractional derivatives ${ }^{c} D_{a^{+}}^{\alpha} x$ and ${ }^{c} D_{b^{-}}^{\alpha} x, a$ is called a left base point and $b$ a right base point. Both $a$ and $b$ are called base points of the fractional derivatives. A fractional differential equation (FDE) containing more than one base point is called a multiple base points FDE ([10]). In this paper, we study the following boundary value problem (BVP) of nonlinear multiple base points fractional differential equations with impulses:

$$
\left\{\begin{array}{l}
{ }^{c} D_{*}^{\alpha} x(t)+\lambda x(t)=f(t, x(t)), \quad t \in J \backslash\left\{t_{1}, t_{2}, \ldots, t_{m-1}\right\}, J=[0,1], \\
\Delta x\left(t_{k}\right)=I_{k}, \quad k=1,2, \ldots, m-1, \\
x(0)+I_{0^{+}}^{\gamma} x(\eta)=0, \quad x(1)+{ }^{c} D_{t_{m}^{+}}^{\delta} x(1)=0, \quad \eta \in\left(0, t_{1}\right),
\end{array}\right.
$$

where $\alpha, \gamma, \delta \in(0,1), \alpha>\gamma, \alpha>\delta, \lambda>0 .{ }^{c} D_{*}^{\cdot}$ is the standard Caputo fractional derivative at the base points $t=t_{k}(k=0,1,2, \ldots, m)$, that is, $\left.{ }^{c} D_{*}^{\cdot}\right|_{\left(t_{k}, t_{k+1}\right]} x(t)={ }^{c} D_{t_{k}^{+}}^{\cdot} x(t)$ for all $t \in\left(t_{k}, t_{k+1}\right]$,

(c) The Author(s) 2017. This article is distributed under the terms of the Creative Commons Attribution 4.0 International License (http://creativecommons.org/licenses/by/4.0/), which permits unrestricted use, distribution, and reproduction in any medium, provided you give appropriate credit to the original author(s) and the source, provide a link to the Creative Commons license, and indicate if changes were made. 
$I_{0^{+}}^{\gamma}$ denotes the fractional integral of order $\gamma, f: J \times \mathbb{R} \rightarrow \mathbb{R}$ is an appropriate function to be specified later. The impulsive moments $\left\{t_{k}\right\}$ are given such that $0<t_{1}<\cdots<t_{m-1}<1$, $\Delta x\left(t_{k}\right)$ represents the jump of function $x$ at $t_{k}$, which is defined by $\Delta x\left(t_{k}\right)=x\left(t_{k}^{+}\right)-x\left(t_{k}^{-}\right)$, where $x\left(t_{k}^{+}\right), x\left(t_{k}^{-}\right)$represent the right and left limits of $x(t)$ at $t=t_{k}$ respectively, the constant $I_{k}$ denotes the size of the jump.

In 1954, Barrett ([6]) applied the method of successive approximations to derive the existence of solutions to the fractional differential equations of order $\alpha \in(0,1)$ with constant coefficients. Recently, as mentioned in $[13,14]$ and the references therein, the existence results of the impulsive fractional differential equations with anti-periodic boundary conditions involving the Caputo differential operator of order $\alpha \in(0,1)$ are obtained by the Mittag-Leffler functions. Inspired by the work of the above papers, the aim of the present paper is to establish some simple criteria for the existence of solutions of BVP (1.1)-(1.3).

The paper is organized as follows. In Section 2, we present some basic concepts, the notations about the fractional calculus and the properties of the Mittag-Leffler functions. In Section 3, we present the definition of solution for (1.1)-(1.3). In Section 4, by applying Krasnoselskii's fixed point theorem, we verify the existence of solutions for problem (1.1)(1.3). We give an example to illustrate the result in Section 5.

\section{Preliminaries}

In this paper, we denote by $L^{p}(J, \mathbb{R})$ the Banach space of all Lebesgue measurable functions $l: J \rightarrow \mathbb{R}$ with the norm $\|l\|_{L^{p}}=\left(\int_{J}|l(t)|^{p} d t\right)^{\frac{1}{p}}<\infty$ and by $\mathrm{AC}([a, b], \mathbb{R})$ the space of all the absolutely continuous functions defined on $[a, b]$.

Definition 2.1 ([2,3]) The fractional integral of order $q$ with the lower limit $a$ for a function $g(t) \in L^{1}([a,+\infty), \mathbb{R})$ is defined as

$$
\left(I_{a^{+}}^{q} g\right)(t)=\frac{1}{\Gamma(q)} \int_{a}^{t}(t-s)^{q-1} g(s) d s, \quad t>a, q>0
$$

where $\Gamma(\cdot)$ is the gamma function.

Definition $2.2([2,3])$ If $g(t) \in \mathrm{AC}^{n}([a, b], \mathbb{R})$, then the Riemann-Liouville fractional derivative $\left({ }^{L} D_{a^{+}}^{q} g\right)(t)$ of order $q$ exists almost everywhere on $[a, b]$ and can be written as

$$
\left({ }^{L} D_{a^{+}}^{q} g\right)(t)=\frac{1}{\Gamma(n-q)} \frac{d^{n}}{d t^{n}} \int_{a}^{t}(t-s)^{n-q-1} g(s) d s, \quad t>a, n-1<q<n .
$$

Definition $2.3([2,3])$ If $g(t) \in \mathrm{AC}^{n}([a, b], \mathbb{R})$, then the Caputo derivative $\left({ }^{c} D_{a^{+}}^{q} g\right)(t)$ of order $q$ exists almost everywhere on $[a, b]$ and can be written as

$$
\left({ }^{c} D_{a^{+}}^{q} g\right)(t)=\left({ }^{L} D_{a^{+}}^{q}\left[g(s)-\sum_{k=0}^{n-1} \frac{g^{(k)}(a)}{k !}(s-a)^{k}\right]\right)(t), \quad t>a, n-1<q<n,
$$

moreover, if $g(a)=g^{\prime}(a)=\cdots=g^{(n-1)}(a)=0$, then $\left({ }^{c} D_{a^{+}}^{q} g\right)(t)=\left({ }^{L} D_{a^{+}}^{q} g\right)(t)$. 
Remark 2.4 ([2, 3]) The Caputo fractional derivative of order $q$ for a function $g \in$ $C^{n}([a, b], \mathbb{R})$ is defined by

$$
\left({ }^{c} D_{a^{+}}^{q} g\right)(t)=\frac{1}{\Gamma(n-q)} \int_{a}^{t} \frac{g^{(n)}(s)}{(t-s)^{q-n+1}} d s, \quad t>a, n-1<q<n .
$$

Definition $2.5([2,3])$ For $\alpha, \beta>0, z \in \mathbb{C}$, the classical Mittag-Leffler function $E_{\alpha}(z)$ and the generalized Mittag-Leffler functions $E_{\alpha, \beta}(z)$ are defined by

$$
\begin{aligned}
& E_{\alpha}(z)=\sum_{k=0}^{\infty} \frac{z^{k}}{\Gamma(\alpha k+1)}, \quad E_{\alpha, \beta}(z)=\sum_{k=0}^{\infty} \frac{z^{k}}{\Gamma(\alpha k+\beta)}, \\
& E_{\alpha, \beta}^{\rho}(z)=\sum_{k=0}^{\infty} \frac{z^{k}}{\Gamma(\alpha k+\beta)} \frac{(\rho)_{k}}{k !}
\end{aligned}
$$

where $(\rho)_{0}=1$ and $(\rho)_{k}=\rho(\rho+1) \cdots(\rho+k-1)$ for $k \in \mathbb{N}$.

Clearly, $E_{\alpha, 1}(z)=E_{\alpha}(z)$.

Lemma 2.6 ([2]) Let $v, \beta, \alpha>0$. The usual derivatives of $E_{\alpha}(z), E_{\alpha, \beta}(z)$ and the RiemannLiouville integration of $E_{\alpha}\left(-\lambda t^{\alpha}\right)$ are expressed by

(1) $\left(\frac{d}{d z}\right)^{n}\left[E_{\alpha, \beta}(z)\right]=n ! E_{\alpha, \beta+\alpha n}^{n+1}(z), n \in \mathbb{N}$;

(2) $\left(\frac{d}{d z}\right)^{n}\left[E_{\alpha}(z)\right]=n ! E_{\alpha, 1+\alpha n}^{n+1}(z), n \in \mathbb{N}$;

(3) $\left(\frac{d}{d t}\right)^{n}\left[t^{\beta-1} E_{\alpha, \beta}\left(-\lambda t^{\alpha}\right)\right]=t^{\beta-n-1} E_{\alpha, \beta-n}\left(-\lambda t^{\alpha}\right), n \geq 1$;

(4) $\left[I_{0^{+}}^{\beta}\left(s^{\nu-1} E_{\alpha, \nu}\left(-\lambda s^{\alpha}\right)\right)\right](t):=\frac{1}{\Gamma(\beta)} \int_{0}^{t}(t-s)^{\beta-1} s^{\nu-1} E_{\alpha, \nu}\left(-\lambda s^{\alpha}\right) d s=t^{\beta+\nu-1} E_{\alpha, \beta+\nu}\left(-\lambda t^{\alpha}\right)$.

As mentioned in ([14]), $E_{\alpha}\left(-\lambda t^{\alpha}\right)$ and $E_{\alpha, \alpha}\left(-\lambda t^{\alpha}\right)$ can be represented by

$$
\begin{aligned}
& E_{\alpha}\left(-\lambda t^{\alpha}\right)=\int_{0}^{\infty} e^{-\lambda t^{\alpha} \theta} \phi(\theta) d \theta, \\
& E_{\alpha, \alpha}\left(-\lambda t^{\alpha}\right)=\alpha \int_{0}^{\infty} \theta e^{-\lambda t^{\alpha} \theta} \phi(\theta) d \theta,
\end{aligned}
$$

where

$$
\phi(\theta)=\frac{1}{\pi} \sum_{n=1}^{\infty}(-1)^{n-1} \theta^{\alpha n-1} \frac{\Gamma(n \alpha+1)}{n !} \sin (n \pi \alpha), \quad 0<\alpha<1, \theta>0 .
$$

Moreover,

$$
\int_{0}^{\infty} \theta^{\xi} \phi(\theta) d \theta=\frac{\Gamma(\xi+1)}{\Gamma(\alpha \xi+1)} \quad(\xi \geq 0)
$$

Lemma 2.7 For $\lambda>0, \alpha, \beta, \theta_{1}, \theta_{2} \in(0,1), \alpha \geq \theta_{2}$, the generalized Mittag-Leffler functions have the following properties:

(1) $\frac{d}{d t}\left[E_{\alpha}\left(-\lambda t^{\alpha}\right)\right]=-\lambda t^{\alpha-1} E_{\alpha, \alpha}\left(-\lambda t^{\alpha}\right)$;

(2) $E_{\alpha, \alpha+\beta}\left(-\lambda t^{\alpha}\right)=\frac{1}{\Gamma(\beta)} \int_{0}^{1} E_{\alpha, \alpha}\left(-\lambda t^{\alpha} u^{\alpha}\right) u^{\alpha-1}(1-u)^{\beta-1} d u$;

(3) $E_{\alpha, \beta}\left(-\lambda t^{\alpha}\right)=\frac{1}{\Gamma(\beta)}-\lambda t^{\alpha} E_{\alpha, \alpha+\beta}\left(-\lambda t^{\alpha}\right)$;

(4) $E_{\alpha, \theta_{1}+1}\left(-\lambda t^{\alpha}\right)=\frac{1}{\Gamma\left(\theta_{1}\right)} \int_{0}^{1} E_{\alpha}\left(-\lambda t^{\alpha} u^{\alpha}\right)(1-u)^{\theta_{1}-1} d u$; 
(5) $\left[{ }^{c} D_{a^{+}}^{\theta_{2}} E_{\alpha}\left(-\lambda(s-a)^{\alpha}\right)\right](t)=-\lambda(t-a)^{\alpha-\theta_{2}} E_{\alpha, \alpha-\theta_{2}+1}\left(-\lambda(t-a)^{\alpha}\right)$.

In particular, when $\alpha=\theta_{2},\left[{ }^{c} D_{a^{+}}^{\alpha} E_{\alpha}\left(-\lambda(s-a)^{\alpha}\right)\right](t)=-\lambda E_{\alpha}\left(-\lambda(t-a)^{\alpha}\right)$.

Proof We denote the beta function by $\mathbb{B}(\cdot, \cdot)$. From Lemma 2.6(2),

$$
\begin{aligned}
\frac{d}{d t}\left[E_{\alpha}\left(-\lambda t^{\alpha}\right)\right] & =-\lambda \alpha t^{\alpha-1} E_{\alpha, 1+\alpha}^{2}\left(-\lambda t^{\alpha}\right) \\
& =-\lambda \alpha t^{\alpha-1} \sum_{k=0}^{\infty} \frac{\left(-\lambda t^{\alpha}\right)^{k}(1+k)}{\Gamma(\alpha k+1+\alpha)} \\
& =-\lambda t^{\alpha-1} \sum_{k=0}^{\infty} \frac{\left(-\lambda t^{\alpha}\right)^{k}}{\Gamma(\alpha k+\alpha)} \\
& =-\lambda t^{\alpha-1} E_{\alpha, \alpha}\left(-\lambda t^{\alpha}\right) .
\end{aligned}
$$

From [14], the second result holds. Moreover,

$$
\begin{aligned}
E_{\alpha, \beta}\left(-\lambda t^{\alpha}\right) & =\sum_{k=0}^{\infty} \frac{\left(-\lambda t^{\alpha}\right)^{k}}{\Gamma(\alpha k+\beta)}=\frac{1}{\Gamma(\beta)}-\lambda t^{\alpha} \sum_{k=1}^{\infty} \frac{\left(-\lambda t^{\alpha}\right)^{k-1}}{\Gamma(\alpha k+\beta)} \\
& =\frac{1}{\Gamma(\beta)}-\lambda t^{\alpha} E_{\alpha, \alpha+\beta}\left(-\lambda t^{\alpha}\right), \\
E_{\alpha, \theta_{1}+1}\left(-\lambda t^{\alpha}\right) & =\sum_{k=0}^{\infty} \frac{\left(-\lambda t^{\alpha}\right)^{k}}{\Gamma\left(\alpha k+\theta_{1}+1\right)}=\frac{1}{\Gamma\left(\theta_{1}\right)} \sum_{k=0}^{\infty} \frac{\left(-\lambda t^{\alpha}\right)^{k} \mathbb{B}\left(\alpha k+1, \theta_{1}\right)}{\Gamma(\alpha k+1)} \\
& =\frac{1}{\Gamma\left(\theta_{1}\right)} \int_{0}^{1} \sum_{k=0}^{\infty} \frac{\left(-\lambda t^{\alpha} u^{\alpha}\right)^{k}}{\Gamma(\alpha k+1)}(1-u)^{\theta_{1}-1} d u \\
& =\frac{1}{\Gamma\left(\theta_{1}\right)} \int_{0}^{1} E_{\alpha}\left(-\lambda t^{\alpha} u^{\alpha}\right)(1-u)^{\theta_{1}-1} d u .
\end{aligned}
$$

Applying Remark 2.4 and the fact $\int_{a}^{t}(t-s)^{m_{1}-1}(s-a)^{m_{2}-1} d s=(t-a)^{m_{1}+m_{2}-1} \mathbb{B}\left(m_{1}, m_{2}\right)$, we have

$$
\begin{aligned}
{\left[{ }^{c} D_{a^{+}}^{\theta_{2}} E_{\alpha}\left(-\lambda(s-a)^{\alpha}\right)\right](t) } & =\frac{1}{\Gamma\left(1-\theta_{2}\right)} \int_{a}^{t}(t-s)^{-\theta_{2}} \frac{d}{d s}\left(\sum_{k=0}^{\infty} \frac{\left.\left(-\lambda(s-a)^{\alpha}\right)^{k}\right)}{\Gamma(\alpha k+1)}\right) d s \\
& =\frac{1}{\Gamma\left(1-\theta_{2}\right)} \sum_{k=1}^{\infty} \frac{(-\lambda)^{k}}{\Gamma(\alpha k)} \int_{a}^{t}(t-s)^{-\theta_{2}}(s-a)^{\alpha k-1} d s \\
& =-\lambda(t-a)^{\alpha-\theta_{2}} \sum_{k=1}^{\infty} \frac{(-\lambda)^{k-1}(t-a)^{\alpha(k-1)}}{\Gamma\left(\alpha k+1-\theta_{2}\right)} \\
& =-\lambda(t-a)^{\alpha-\theta_{2}} E_{\alpha, \alpha-\theta_{2}+1}\left(-\lambda(t-a)^{\alpha}\right) .
\end{aligned}
$$

Lemma 2.8 ([3]) If $0<\alpha<2, \beta$ is an arbitrary real number, $\frac{\pi \alpha}{2}<\mu<\min \{\pi, \pi \alpha\}$, then

$$
\left|E_{\alpha, \beta}(z)\right| \leq \frac{\mathcal{C}}{1+|z|}, \quad \mu \leq|\arg (z)| \leq \pi, \quad|z| \geq 0,
$$

where $\mathcal{C}$ is a positive constant. 
Lemma 2.9 Let $\alpha, \beta \in(0,1), \lambda>0$. Then the functions $E_{\alpha}, E_{\alpha, \alpha}$ and $E_{\alpha, \alpha+\beta}$ are nonnegative and have the following properties:

(i) For any $t \in J, E_{\alpha}\left(-\lambda t^{\alpha}\right) \leq 1, E_{\alpha, \alpha}\left(-\lambda t^{\alpha}\right) \leq \frac{1}{\Gamma(\alpha)}, E_{\alpha, \alpha+\beta}\left(-\lambda t^{\alpha}\right) \leq \frac{1}{\Gamma(\alpha+\beta)}$,

$E_{\alpha, \beta}\left(-\lambda t^{\alpha}\right) \leq \frac{1}{\Gamma(\beta)}$, moreover, $E_{\alpha}(0)=1$. In particular,

$$
E_{\alpha, \alpha-\delta}\left(-\lambda t^{\alpha}\right) \leq \frac{1}{\Gamma(\alpha-\delta)}, \quad\left|E_{\alpha, \alpha-\delta}\left(-\lambda t^{\alpha}\right)\right| \leq \mathcal{C} .
$$

(ii) For any $t_{1}, t_{2} \in J$,

$$
\begin{aligned}
& \left|E_{\alpha}\left(-\lambda t_{2}^{\alpha}\right)-E_{\alpha}\left(-\lambda t_{1}^{\alpha}\right)\right|=O\left(\left|t_{2}-t_{1}\right|^{\alpha}\right), \quad \text { as } t_{2} \rightarrow t_{1}, \\
& \left|E_{\alpha, \alpha}\left(-\lambda t_{2}^{\alpha}\right)-E_{\alpha, \alpha}\left(-\lambda t_{1}^{\alpha}\right)\right|=O\left(\left|t_{2}-t_{1}\right|^{\alpha}\right), \quad \text { as } t_{2} \rightarrow t_{1}, \\
& \left|E_{\alpha, \alpha-\delta}\left(-\lambda t_{2}^{\alpha}\right)-E_{\alpha, \alpha-\delta}\left(-\lambda t_{1}^{\alpha}\right)\right|=O\left(\left|t_{2}-t_{1}\right|^{\alpha}\right), \quad \text { as } t_{2} \rightarrow t_{1} .
\end{aligned}
$$

Proof (i) From (2.1), we get $E_{\alpha}\left(-\lambda t^{\alpha}\right)=\int_{0}^{\infty} e^{-\lambda t^{\alpha} \theta} \phi(\theta) d \theta \leq \int_{0}^{\infty} \phi(\theta) d \theta=1$.

By (2.2), we find $E_{\alpha, \alpha}\left(-\lambda t^{\alpha}\right)=\alpha \int_{0}^{\infty} \theta e^{-\lambda t^{\alpha} \theta} \phi(\theta) d \theta \leq \frac{1}{\Gamma(\alpha)}$.

Using Lemma 2.7(2), one sees

$$
E_{\alpha, \alpha+\beta}\left(-\lambda t^{\alpha}\right)=\frac{1}{\Gamma(\beta)} \int_{0}^{1} E_{\alpha, \alpha}\left(-\lambda t^{\alpha} u^{\alpha}\right) u^{\alpha-1}(1-u)^{\beta-1} d u \leq \frac{1}{\Gamma(\alpha+\beta)} .
$$

Noting $E_{\alpha, \alpha+\beta}\left(-\lambda t^{\alpha}\right)>0$ and Lemma 2.7(3), we have $E_{\alpha, \beta}\left(-\lambda t^{\alpha}\right) \leq \frac{1}{\Gamma(\beta)}$. Taking $\beta=$ $\alpha-\delta$ in $E_{\alpha, \beta}\left(-\lambda t^{\alpha}\right) \leq \frac{1}{\Gamma(\beta)}$, we obtain $E_{\alpha, \alpha-\delta}\left(-\lambda t^{\alpha}\right) \leq \frac{1}{\Gamma(\alpha-\delta)}$. By Lemma 2.8, we get $\left|E_{\alpha, \alpha-\delta}\left(-\lambda t^{\alpha}\right)\right| \leq \mathcal{C}$.

(ii) For $0 \leq t_{1}<t_{2} \leq 1$, using the Lagrange mean value theorem and the fact $\left|t_{2}^{\alpha}-t_{1}^{\alpha}\right| \leq$ $\left(t_{2}-t_{1}\right)^{\alpha},(2.1),(2.2)$ and (2.3), we find

$$
\begin{aligned}
\left|E_{\alpha}\left(-\lambda t_{2}^{\alpha}\right)-E_{\alpha}\left(-\lambda t_{1}^{\alpha}\right)\right| & =\int_{0}^{\infty}\left|e^{-\lambda t_{2}^{\alpha} \theta}-e^{-\lambda t_{1}^{\alpha} \theta}\right| \phi(\theta) d \theta \leq \lambda\left(t_{2}-t_{1}\right)^{\alpha} \int_{0}^{\infty} \theta \phi(\theta) d \theta \\
& =\frac{\lambda\left(t_{2}-t_{1}\right)^{\alpha}}{\Gamma(\alpha+1)}:=O\left(\left|t_{2}-t_{1}\right|^{\alpha}\right), \quad \text { as } t_{2} \rightarrow t_{1}, \\
\left|E_{\alpha, \alpha}\left(-\lambda t_{2}^{\alpha}\right)-E_{\alpha, \alpha}\left(-\lambda t_{1}^{\alpha}\right)\right| & =\alpha \int_{0}^{\infty}\left|e^{-\lambda t_{2}^{\alpha} \theta}-e^{-\lambda t_{1}^{\alpha} \theta}\right| \theta \phi(\theta) d \theta \\
& \leq \frac{2 \lambda \alpha\left(t_{2}-t_{1}\right)^{\alpha}}{\Gamma(2 \alpha+1)}:=O\left(\left|t_{2}-t_{1}\right|^{\alpha}\right), \quad \text { as } t_{2} \rightarrow t_{1},
\end{aligned}
$$

by Lemma 2.7(3), Lemma 2.9(i) and Lemma 2.7(2), one has

$$
\begin{aligned}
&\left|E_{\alpha, \alpha-\delta}\left(-\lambda t_{2}^{\alpha}\right)-E_{\alpha, \alpha-\delta}\left(-\lambda t_{1}^{\alpha}\right)\right| \\
&=\lambda\left|t_{2}^{\alpha} E_{\alpha, 2 \alpha-\delta}\left(-\lambda t_{2}^{\alpha}\right)-t_{1}^{\alpha} E_{\alpha, 2 \alpha-\delta}\left(-\lambda t_{1}^{\alpha}\right)\right| \\
& \leq \lambda\left[\left|t_{2}-t_{1}\right|^{\alpha} E_{\alpha, 2 \alpha-\delta}\left(-\lambda t_{2}^{\alpha}\right)+t_{1}^{\alpha}\left|E_{\alpha, 2 \alpha-\delta}\left(-\lambda t_{2}^{\alpha}\right)-E_{\alpha, 2 \alpha-\delta}\left(-\lambda t_{1}^{\alpha}\right)\right|\right] \\
& \leq \frac{\lambda}{\Gamma(2 \alpha-\delta)}\left|t_{2}-t_{1}\right|^{\alpha} \\
&+\frac{\lambda}{\Gamma(\alpha-\delta)} \int_{0}^{1}\left|E_{\alpha, \alpha}\left(-\lambda t_{2}^{\alpha} u^{\alpha}\right)-E_{\alpha, \alpha}\left(-\lambda t_{1}^{\alpha} u^{\alpha}\right)\right| u^{\alpha-1}(1-u)^{\alpha-\delta-1} d u \\
&:= O\left(\left|t_{2}-t_{1}\right|^{\alpha}\right), \quad \text { as } t_{2} \rightarrow t_{1} .
\end{aligned}
$$


Lemma 2.10 ([2]) The solution to the Cauchy problem

$$
\left\{\begin{array}{l}
{ }^{c} D_{a^{+}}^{\alpha} x(t)+\lambda x(t)=f(t), \\
x(a)=b_{1}, \quad b_{1} \in \mathbb{R}
\end{array}\right.
$$

with $0<\alpha<1$ has the form

$$
x(t)=b_{1} E_{\alpha}\left(-\lambda(t-a)^{\alpha}\right)+\int_{a}^{t}(t-s)^{\alpha-1} E_{\alpha, \alpha}\left(-\lambda(t-s)^{\alpha}\right) f(s) d s
$$

Theorem 2.11 (Krasnoselskii's fixed point theorem) Let $\mathcal{M}$ be a closed convex and nonempty subset of a Banach space X. Let $\mathcal{A}, \mathcal{B}$ be two operators such that (i) $\mathcal{A} x+\mathcal{B} y \in \mathcal{M}$ whenever $x, y \in \mathcal{M}$, (ii) $\mathcal{A}$ is compact and continuous, (iii) $\mathcal{B}$ is a contraction mapping. Then there exists a $z \in \mathcal{M}$ such that $z=\mathcal{A} z+\mathcal{B} z$.

\section{Solutions for BVP}

Setting $J_{0}=\left[0, t_{1}\right], J_{k}=\left(t_{k}, t_{k+1}\right], k=1, \ldots, m-1, J_{m}=\left[t_{m}, 1\right]$, and we define $X=\{x:[0,1] \rightarrow$ $\mathbb{R}:\left.x\right|_{J_{k}} \in C\left(J_{k}, \mathbb{R}\right)$ and there exist $x\left(t_{k}^{+}\right)$and $x\left(t_{k}^{-}\right)$, with $\left.x\left(t_{k}^{-}\right)=x\left(t_{k}\right), k=1, \ldots, m-1\right\}$ with the norm

$$
\|x\|_{1}:=\sup _{k=0,1, \ldots, m} \sup _{t \in J_{k}}|x(t)|
$$

Obviously, $X$ is a real Banach space.

In this paper, we consider the following assumption.

(H1) $f: J \times \mathbb{R} \rightarrow \mathbb{R}$ satisfies $f(\cdot, x): J \rightarrow \mathbb{R}$ is measurable for all $x \in \mathbb{R}$ and $f(t, \cdot): \mathbb{R} \rightarrow \mathbb{R}$ is continuous for a.e. $t \in J$, and there exists a function $\mu \in L^{\frac{1}{q_{1}}}\left(J, \mathbb{R}^{+}\right)\left(0<q_{1}<\min \left\{\frac{\alpha}{2}, \alpha-\right.\right.$ $\delta\})$ such that $|f(t, x)| \leq \mu(t)$.

Definition 3.1 A function $x: J \rightarrow \mathbb{R}$ is said to be a solution of (1.1)-(1.3) if

(1) $x \in \mathrm{AC}\left(J_{k}, \mathbb{R}\right)$;

(2) $x$ satisfies the equation ${ }^{c} D_{t^{+}}^{\alpha} x(t)+\lambda x(t)=f(t, x(t))$ on $J_{k}$;

(3) for $k=1,2, \ldots, m-1, \Delta x\left(t_{k}\right)=I_{k}$, and $x(0)+I_{0^{+}}^{\gamma} x(\eta)=0, x(1)+{ }^{c} D_{t_{m}^{+}}^{\delta} x(1)=0$.

Next, we present the following lemmas.

Lemma 3.2 For any $\tau_{2}, \tau_{1} \in J_{k}(k=0,1,2, \ldots, m)$ and $\tau_{2}<\tau_{1}$,

$$
\int_{t_{k}}^{\tau_{2}}\left[\left(\tau_{2}-s\right)^{\alpha-1}-\left(\tau_{1}-s\right)^{\alpha-1}\right] \mu(s) d s \rightarrow 0, \quad \text { as } \tau_{2} \rightarrow \tau_{1} .
$$

Proof It follows from the Hölder inequality that

$$
\begin{aligned}
& \left|\int_{t_{k}}^{\tau_{2}}\left[\left(\tau_{2}-s\right)^{\alpha-1}-\left(\tau_{1}-s\right)^{\alpha-1}\right] \mu(s) d s\right| \\
& \quad \leq\|\mu\|_{L^{\frac{1}{q_{1}}}}\left[\int_{t_{k}}^{\tau_{2}}\left|\left(\tau_{2}-s\right)^{\alpha-1}-\left(\tau_{1}-s\right)^{\alpha-1}\right|^{\frac{1}{1-q_{1}}} d s\right]^{1-q_{1}}
\end{aligned}
$$




$$
\begin{aligned}
& =(1-\alpha)\|\mu\|_{L^{\frac{1}{q_{1}}}}\left(\int_{t_{k}}^{\tau_{2}}\left|\int_{\tau_{2}}^{\tau_{1}}(\zeta-s)^{\alpha-2} d \zeta\right|^{\frac{1}{1-q_{1}}} d s\right)^{1-q_{1}} \\
& \leq \bar{M}\left[\int_{t_{k}}^{\tau_{2}}\left(\left(\tau_{2}-s\right)^{\theta}-\left(\tau_{1}-s\right)^{\theta}\right) d s\right]^{1-q_{1}} \\
& =\frac{\bar{M}}{(1+\theta)^{1-q_{1}}}\left[\left(\tau_{1}-\tau_{2}\right)^{1+\theta}-\left(\tau_{1}-t_{k}\right)^{1+\theta}+\left(\tau_{2}-t_{k}\right)^{1+\theta}\right]^{1-q_{1}} \\
& \rightarrow 0, \quad \text { as } \tau_{2} \rightarrow \tau_{1},
\end{aligned}
$$

where $\bar{M}>0$ is a constant and $\theta=\frac{\alpha-1-q_{1}}{1-q_{1}} \in(-1,0)$.

For $y>q_{1}$ and $t_{i-1} \in J(i=1, \ldots, m+1)$, from the Hölder inequality, we have

$$
\int_{t_{i-1}}^{t_{i}}\left(t_{i}-s\right)^{y-1} \mu(s) d s \leq\left(\int_{t_{i-1}}^{t_{i}}\left(t_{i}-s\right)^{\frac{y-1}{1-q_{1}}} d s\right)^{1-q_{1}}\|\mu\|_{L^{\frac{1}{q_{1}}}}=\zeta_{y}\left(t_{i}-t_{i-1}\right)^{y-q_{1}}
$$

where $\zeta_{y}=\left(\frac{1-q_{1}}{y-q_{1}}\right)^{1-q_{1}}\|\mu\|_{L} \frac{1}{q_{1}}$.

For brevity, we define

$$
\left(Q_{k}^{\varsigma} x\right)(t):=\int_{t_{k}}^{t}(t-s)^{s^{-1}} E_{\alpha, \zeta}\left(-\lambda(t-s)^{\alpha}\right) f(s, x(s)) d s
$$

then, for $t \in\left(t_{k}, t_{k+1}\right]$, from (3.1) and Lemma 2.9(i), we obtain

$$
\begin{aligned}
& \left|\left(Q_{k}^{\alpha} x\right)(t)\right| \leq \int_{t_{k}}^{t} \frac{(t-s)^{\alpha-1} \mu(s)}{\Gamma(\alpha)} d s \leq \zeta_{\alpha} \frac{\left(t-t_{k}\right)^{\alpha-q_{1}}}{\Gamma(\alpha)} \\
& \left|\left(Q_{k}^{\alpha-\delta} x\right)(t)\right| \leq \mathcal{C} \int_{t_{k}}^{t}(t-s)^{\alpha-\delta-1} \mu(s) d s \leq \mathcal{C} \zeta_{\alpha-\delta}\left(t-t_{k}\right)^{\alpha-\delta-q_{1}},
\end{aligned}
$$

which means that $(t-s)^{\alpha-1} E_{\alpha, \alpha}\left(-\lambda(t-s)^{\alpha}\right) f(s, x(s))$ and $(t-s)^{\alpha-\delta-1} E_{\alpha, \alpha-\delta}\left(-\lambda(t-s)^{\alpha}\right) f(s, x(s))$ are Lebesgue integrable with respect to $s \in\left[t_{k}, t_{k+1}\right]$ for all $t \in\left[t_{k}, t_{k+1}\right]$ and $x \in X$.

Lemma 3.3 For any $k=0,1,2, \ldots, m,\left(Q_{k}^{\alpha} x\right)(t) \in C\left(J_{k}, \mathbb{R}\right),\left(Q_{k}^{\alpha-\delta} x\right)(t) \in C\left(J_{k}, \mathbb{R}\right)$.

Proof For any $h>0, t_{k}<t<t+h<t_{k+1}$, by (H1), Lemma 2.9(i), (ii), Lemma 3.2 and (3.1), we get

$$
\begin{aligned}
& \left|\left(Q_{k}^{\alpha} x\right)(t+h)-\left(Q_{k}^{\alpha} x\right)(t)\right| \\
& \leq \int_{t_{k}}^{t}\left|(t+h-s)^{\alpha-1}-(t-s)^{\alpha-1}\right| E_{\alpha, \alpha}\left(-\lambda(t+h-s)^{\alpha}\right)|f(s, x(s))| d s \\
& \quad+\int_{t_{k}}^{t}(t-s)^{\alpha-1}\left|E_{\alpha, \alpha}\left(-\lambda(t+h-s)^{\alpha}\right)-E_{\alpha, \alpha}\left(-\lambda(t-s)^{\alpha}\right)\right||f(s, x(s))| d s \\
& \quad+\int_{t}^{t+h}(t+h-s)^{\alpha-1} E_{\alpha, \alpha}\left(-\lambda(t+h-s)^{\alpha}\right)|f(s, x(s))| d s \\
& \leq \int_{t_{k}}^{t} \frac{\left|(t+h-s)^{\alpha-1}-(t-s)^{\alpha-1}\right|}{\Gamma(\alpha)} \mu(s) d s+O\left(h^{\alpha}\right) \int_{t_{k}}^{t}(t-s)^{\alpha-1} \mu(s) d s
\end{aligned}
$$




$$
\begin{aligned}
& +\int_{t}^{t+h} \frac{(t+h-s)^{\alpha-1}}{\Gamma(\alpha)} \mu(s) d s \\
& \rightarrow 0, \quad \text { as } h \rightarrow 0 .
\end{aligned}
$$

Similarly, noting (2.4) and (2.5), we find $\left(Q_{k}^{\alpha-\delta} x\right)(t) \in C\left(J_{k}, \mathbb{R}\right)$.

Lemma 3.4 Assume that (H1) holds. Then $\left(Q_{k}^{\alpha} x\right)(t) \in \mathrm{AC}\left(\left[t_{k}, t_{k+1}\right], \mathbb{R}\right)$, for $x \in X, k=$ $0,1, \ldots, m$.

Proof For every finite collection $\left\{\left(a_{i}, b_{i}\right)\right\}_{1 \leq i \leq n}$ on $\left[t_{k}, t_{k+1}\right]$ with $\sum_{i=1}^{n}\left(b_{i}-a_{i}\right) \rightarrow 0$, noting (3.1), Lemma 3.2 and Lemma 2.9(ii), we have

$$
\begin{aligned}
\sum_{i=1}^{n} \mid & \left(Q_{k}^{\alpha} x\right)\left(b_{i}\right)-\left(Q_{k}^{\alpha} x\right)\left(a_{i}\right) \mid \\
\leq & \sum_{i=1}^{n}\left|\int_{a_{i}}^{b_{i}}\left(b_{i}-s\right)^{\alpha-1} E_{\alpha, \alpha}\left(-\lambda\left(b_{i}-s\right)^{\alpha}\right) f(s, x(s)) d s\right| \\
& +\sum_{i=1}^{n} \int_{t_{k}}^{a_{i}}\left|\left[\left(b_{i}-s\right)^{\alpha-1}-\left(a_{i}-s\right)^{\alpha-1}\right] E_{\alpha, \alpha}\left(-\lambda\left(b_{i}-s\right)^{\alpha}\right) f(s, x(s))\right| d s \\
& +\sum_{i=1}^{n} \int_{t_{k}}^{a_{i}}\left(a_{i}-s\right)^{\alpha-1}\left|E_{\alpha, \alpha}\left(-\lambda\left(b_{i}-s\right)^{\alpha}\right)-E_{\alpha, \alpha}\left(-\lambda\left(a_{i}-s\right)^{\alpha}\right)\right||f(s, x(s))| d s \\
\leq & \sum_{i=1}^{n} \int_{a_{i}}^{b_{i}} \frac{\left(b_{i}-s\right)^{\alpha-1} \mu(s)}{\Gamma(\alpha)} d s+\frac{1}{\Gamma(\alpha)} \sum_{i=1}^{n} \int_{t_{k}}^{a_{i}}\left[\left(a_{i}-s\right)^{\alpha-1}-\left(b_{i}-s\right)^{\alpha-1}\right] \mu(s) d s \\
& +\sum_{i=1}^{n} \int_{t_{k}}^{a_{i}}\left(a_{i}-s\right)^{\alpha-1} \mu(s) d s \cdot O\left(\left|b_{i}-a_{i}\right|^{\alpha}\right) \\
\leq & \frac{\zeta_{\alpha}}{\Gamma(\alpha)} \sum_{i=1}^{n}\left(b_{i}-a_{i}\right)^{\alpha-q_{1}}+\frac{1}{\Gamma(\alpha)} \sum_{i=1}^{n} \int_{t_{k}}^{a_{i}}\left[\left(a_{i}-s\right)^{\alpha-1}-\left(b_{i}-s\right)^{\alpha-1}\right] \mu(s) d s \\
& +\zeta_{\alpha} \sum_{i=1}^{n} O\left(\left|b_{i}-a_{i}\right|^{\alpha}\right) \\
\rightarrow & 0 .
\end{aligned}
$$

Hence, $\left(Q_{k}^{\alpha} x\right)(t)$ is absolutely continuous on $\left[t_{k}, t_{k+1}\right]$. Furthermore, for almost all $t \in$ $\left[t_{k}, t_{k+1}\right],\left[{ }^{c} D_{t_{k}^{+}}^{\alpha}\left(Q_{k}^{\alpha} x\right)(s)\right](t)$ and $\left[{ }^{c} D_{t_{k}^{+}}^{\delta}\left(Q_{k}^{\alpha} x\right)(s)\right](t)$ exist.

Lemma 3.5 Assume that (H1) holds. Then, for $x \in X, k=0,1, \ldots, m$,

$$
\begin{aligned}
& {\left[{ }^{c} D_{t_{k}^{+}}^{\alpha}\left(Q_{k}^{\alpha} x\right)(s)\right](t)=f(t, x(t))-\lambda\left(Q_{k}^{\alpha} x\right)(t), \quad \text { a.e. } t \in J_{k},} \\
& {\left[{ }^{c} D_{t_{k}^{+}}^{\delta}\left(Q_{k}^{\alpha} x\right)(s)\right](t)=\left(Q_{k}^{\alpha-\delta} x\right)(t), \quad \text { a.e. } t \in J_{k} .}
\end{aligned}
$$


Proof According to Lemma 2.6(4), we can see that

$$
\begin{aligned}
\int_{s}^{t}(t-\tau)^{-\alpha}(\tau-s)^{\alpha-1} E_{\alpha, \alpha}\left(-\lambda(\tau-s)^{\alpha}\right) d \tau & =\int_{0}^{t-s}(t-s-\tau)^{-\alpha} \tau^{\alpha-1} E_{\alpha, \alpha}\left(-\lambda \tau^{\alpha}\right) d \tau \\
& =\Gamma(1-\alpha) E_{\alpha}\left(-\lambda(t-s)^{\alpha}\right), \\
\int_{s}^{t}(t-\tau)^{-\delta}(\tau-s)^{\alpha-1} E_{\alpha, \alpha}\left(-\lambda(\tau-s)^{\alpha}\right) d \tau & =\int_{0}^{t-s}(t-s-\tau)^{-\delta} \tau^{\alpha-1} E_{\alpha, \alpha}\left(-\lambda \tau^{\alpha}\right) d \tau \\
& =\Gamma(1-\delta)(t-s)^{\alpha-\delta} E_{\alpha, \alpha-\delta+1}\left(-\lambda(t-s)^{\alpha}\right) .
\end{aligned}
$$

Moreover, noting Lemma 2.6(1) and Lemma 2.7(1), we obtain

$$
\begin{aligned}
& {\left[{ }^{L} D_{t_{k}^{+}}^{\alpha}\left(Q_{k}^{\alpha} x\right)(s)\right](t)} \\
& \quad=\frac{1}{\Gamma(1-\alpha)} \frac{d}{d t} \int_{t_{k}}^{t}(t-s)^{-\alpha}\left[\int_{t_{k}}^{s}(s-\tau)^{\alpha-1} E_{\alpha, \alpha}\left(-\lambda(s-\tau)^{\alpha}\right) f(\tau, x(\tau)) d \tau\right] d s \\
& \quad=\frac{1}{\Gamma(1-\alpha)} \frac{d}{d t} \int_{t_{k}}^{t} f(\tau, x(\tau)) d \tau \int_{\tau}^{t}(t-s)^{-\alpha}(s-\tau)^{\alpha-1} E_{\alpha, \alpha}\left(-\lambda(s-\tau)^{\alpha}\right) d \tau \\
& \quad=\frac{d}{d t} \int_{t_{k}}^{t} E_{\alpha}\left(-\lambda(t-\tau)^{\alpha}\right) f(\tau, x(\tau)) d \tau \\
& \quad=f(t, x(t))-\lambda\left(Q_{k}^{\alpha} x\right)(t), \quad \text { a.e. } t \in\left[t_{k}, t_{k+1}\right],
\end{aligned}
$$

and by Lemma 2.6(3), one gets

$$
\begin{aligned}
& {\left[{ }^{L} D_{t_{k}^{+}}^{\delta}\left(Q_{k}^{\alpha} x\right)(s)\right](t)} \\
& \quad=\frac{1}{\Gamma(1-\delta)} \frac{d}{d t} \int_{t_{k}}^{t} f(\tau, x(\tau)) d \tau \int_{\tau}^{t}(t-s)^{-\delta}(s-\tau)^{\alpha-1} E_{\alpha, \alpha}\left(-\lambda(s-\tau)^{\alpha}\right) d s \\
& \quad=\frac{d}{d t} \int_{t_{k}}^{t}(t-\tau)^{\alpha-\delta} E_{\alpha, \alpha-\delta+1}\left(-\lambda(t-\tau)^{\alpha}\right) f(\tau, x(\tau)) d \tau \\
& =\int_{t_{k}}^{t}(t-\tau)^{\alpha-\delta-1} E_{\alpha, \alpha-\delta}\left(-\lambda(t-\tau)^{\alpha}\right) f(\tau, x(\tau)) d \tau \\
& =\left(Q_{k}^{\alpha-\delta} x\right)(t), \quad \text { a.e. } t \in\left[t_{k}, t_{k+1}\right] .
\end{aligned}
$$

Noting (3.2) and (3.3), we have $\left(Q_{k}^{\alpha} x\right)\left(t_{k}^{+}\right)=0$ and $\left(Q_{k}^{\alpha-\delta} x\right)\left(t_{k}^{+}\right)=0$. Then, from Definition 2.3, with $g(t)$ replaced by $\left(Q_{k}^{\alpha} x\right)(t)$ and $\left(Q_{k}^{\alpha-\delta} x\right)(t)$, and applying (3.4) and (3.5), we derive

$$
\left[{ }^{c} D_{t_{k}^{+}}^{\alpha}\left(Q_{k}^{\alpha} x\right)(s)\right](t)=\left[{ }^{L} D_{t_{k}^{+}}^{\alpha}\left(Q_{k}^{\alpha} x\right)(s)\right](t)=f(t, x(t))-\lambda\left(Q_{k}^{\alpha} x\right)(t)
$$

and $\left[{ }^{c} D_{t_{k}^{+}}^{\delta}\left(Q_{k}^{\alpha} x\right)(s)\right](t)=\left(Q_{k}^{\alpha-\delta} x\right)(t)$. This completes the proof.

Lemma 3.6 Assume that (H1) holds. Then $\left[I_{0^{+}}^{\gamma}\left(Q_{0}^{\alpha} x\right)(s)\right](t)=\left(Q_{0}^{\alpha+\gamma} x\right)(t)$. 
Proof It follows from (3.2) that $\left(Q_{0}^{\alpha} x\right)(t)$ is Lebesgue integrable, noting Lemma 2.6(4), we have

$$
\begin{aligned}
& {\left[I_{0^{+}}^{\gamma}\left(Q_{0}^{\alpha} x\right)(s)\right](t)} \\
& \quad=\frac{1}{\Gamma(\gamma)} \int_{0}^{t}(t-s)^{\gamma-1}\left(\int_{0}^{s}(s-\tau)^{\alpha-1} E_{\alpha, \alpha}\left(-\lambda(s-\tau)^{\alpha}\right) f(\tau, x(\tau)) d \tau\right) d s \\
& \quad=\frac{1}{\Gamma(\gamma)} \int_{0}^{t} f(\tau, x(\tau)) d \tau \int_{0}^{t-\tau}(t-\tau-s)^{\gamma-1} s^{\alpha-1} E_{\alpha, \alpha}\left(-\lambda s^{\alpha}\right) d s \\
& \quad=\int_{0}^{t}(t-\tau)^{\alpha+\gamma-1} E_{\alpha, \alpha+\gamma}\left(-\lambda(t-\tau)^{\alpha}\right) f(\tau, x(\tau)) d \tau=\left(Q_{0}^{\alpha+\gamma} x\right)(t) .
\end{aligned}
$$

As a consequence of Lemmas 3.4-3.6, by directly computation, we get the following result. For brevity, we define

$$
\begin{aligned}
& \widetilde{c}:=-\frac{\left(Q_{0}^{\alpha+\gamma} x\right)(\eta)}{1+\eta^{\gamma} E_{\alpha, \gamma+1}\left(-\lambda \eta^{\alpha}\right)}, \\
& \left(P_{0} x\right)(t):=\widetilde{c} E_{\alpha}\left(-\lambda t^{\alpha}\right), \\
& \left(P_{i} x\right)(t):=\left[\left(P_{i-1} x\right)\left(t_{i}\right)+\left(Q_{i-1}^{\alpha} x\right)\left(t_{i}\right)+I_{i}\right] E_{\alpha}\left(-\lambda\left(t-t_{i}\right)^{\alpha}\right), \quad i=1, \ldots, m-1, \\
& \left(P_{m} x\right)(t):=-\frac{\left[\left(Q_{m}^{\alpha} x\right)(1)+\left(Q_{m}^{\alpha-\delta} x\right)(1)\right] E_{\alpha}\left(-\lambda\left(t-t_{m}\right)^{\alpha}\right)}{E_{\alpha}\left(-\lambda\left(1-t_{m}\right)^{\alpha}\right)-\lambda\left(1-t_{m}\right)^{\alpha-\delta} E_{\alpha, \alpha-\delta+1}\left(-\lambda\left(1-t_{m}\right)^{\alpha}\right)} .
\end{aligned}
$$

Lemma 3.7 A function $x$ is a solution of (1.1)-(1.3) if and only if $x$ is a solution of the following equation:

$$
x(t)= \begin{cases}\left(P_{0} x\right)(t)+\left(Q_{0}^{\alpha} x\right)(t), & \text { for } t \in J_{0}, \\ \left(P_{1} x\right)(t)+\left(Q_{1}^{\alpha} x\right)(t), & \text { for } t \in J_{1}, \\ \cdots & \\ \left(P_{m-1} x\right)(t)+\left(Q_{m-1}^{\alpha} x\right)(t), & \text { for } t \in J_{m-1}, \\ \left(P_{m} x\right)(t)+\left(Q_{m}^{\alpha} x\right)(t), & \text { for } t \in J_{m} .\end{cases}
$$

Proof (Necessity) For $t \in J_{0}$, it follows from Lemma 2.10 that $x(t)=a_{0} E_{\alpha}\left(-\lambda t^{\alpha}\right)+\left(Q_{0}^{\alpha} x\right)(t)$. Obviously, $x(0)=a_{0}$. Moreover, from Lemma 2.6(4) (taking $\beta:=\gamma, v:=1$ ) and Lemma 3.6, we have

$$
I_{0^{+}}^{\gamma} x(\eta)=a_{0} \eta^{\gamma} E_{\alpha, \gamma+1}\left(-\lambda \eta^{\alpha}\right)+\left(Q_{0}^{\alpha+\gamma} x\right)(\eta) .
$$

Using the condition $x(0)+I_{0^{+}}^{\gamma} x(\eta)=0$, we obtain $a_{0}=\widetilde{c}$, then, for $t \in J_{0}$,

$$
x(t)=\left(P_{0} x\right)(t)+\left(Q_{0}^{\alpha} x\right)(t)
$$

For $t \in J_{1}, x(t)=a_{1} E_{\alpha}\left(-\lambda\left(t-t_{1}\right)^{\alpha}\right)+\left(Q_{1}^{\alpha} x\right)(t)$, since $x\left(t_{1}^{+}\right)=a_{1}=\left(P_{0} x\right)\left(t_{1}\right)+\left(Q_{0}^{\alpha} x\right)\left(t_{1}\right)+I_{1}$, then, for $t \in J_{1}$,

$$
x(t)=\left(P_{1} x\right)(t)+\left(Q_{1}^{\alpha} x\right)(t)
$$


Repeating the above process, we find

$$
x(t)=\left(P_{k} x\right)(t)+\left(Q_{k}^{\alpha} x\right)(t), \quad t \in J_{k}, k=0,1, \ldots, m-1 .
$$

For $t \in J_{m}=\left[t_{m}, 1\right], x(t)=a_{m} E_{\alpha}\left(-\lambda\left(t-t_{m}\right)^{\alpha}\right)+\left(Q_{m}^{\alpha} x\right)(t)$.

Noting Lemma 2.7(5) and Lemma 3.5, we get

$$
{ }^{c} D_{t_{m}^{+}}^{\delta} x(t)=-\lambda a_{m}\left(t-t_{m}\right)^{\alpha-\delta} E_{\alpha, \alpha-\delta+1}\left(-\lambda\left(t-t_{m}\right)^{\alpha}\right)+\left(Q_{m}^{\alpha-\delta} x\right)(t) .
$$

From $x(1)+{ }^{c} D_{t_{m}^{+}}^{\delta} x(1)=0$, one can obtain

$$
a_{m}=-\frac{\left(Q_{m}^{\alpha} x\right)(1)+\left(Q_{m}^{\alpha-\delta} x\right)(1)}{E_{\alpha}\left(-\lambda\left(1-t_{m}\right)^{\alpha}\right)-\lambda\left(1-t_{m}\right)^{\alpha-\delta} E_{\alpha, \alpha-\delta+1}\left(-\lambda\left(1-t_{m}\right)^{\alpha}\right)} .
$$

Now, $x(t)=\left(P_{m} x\right)(t)+\left(Q_{m}^{\alpha} x\right)(t)$.

(Sufficiency) Let $x(t)$ satisfy (3.6). Noting Lemma 2.7(5) and Lemma 3.5, $\left({ }^{c} D_{t^{+}}^{\alpha} x\right)(t)$ exists and ${ }^{c} D_{t_{k}^{+}}^{\alpha} x(t)+\lambda x(t)=f(t, x(t))$ for $t \in J_{k}(k=0,1, \ldots, m)$. Moreover, for $k=1,2, \ldots, m-1$,

$$
\begin{aligned}
x\left(t_{k}^{+}\right)-x\left(t_{k}^{-}\right) & =\left(P_{k} x\right)\left(t_{k}\right)+\left(Q_{k}^{\alpha} x\right)\left(t_{k}\right)-\left(P_{k-1} x\right)\left(t_{k}\right)-\left(Q_{k-1}^{\alpha} x\right)\left(t_{k}\right) \\
& =\left(P_{k-1} x\right)\left(t_{k}\right)+\left(Q_{k-1}^{\alpha} x\right)\left(t_{k}\right)+I_{k}-\left(P_{k-1} x\right)\left(t_{k}\right)-\left(Q_{k-1}^{\alpha} x\right)\left(t_{k}\right) \\
& =I_{k} .
\end{aligned}
$$

The boundary conditions of (1.3) are clearly satisfied, that is, $x(t)$ satisfies (1.1)-(1.3).

\section{Existence result}

In this section, we deal with the existence of solution for the problem (1.1)-(1.3). To this end, we consider the following assumption.

(H2) There exists a function $\psi \in L^{\frac{1}{q_{2}}}\left(J, \mathbb{R}^{+}\right)\left(q_{2} \in(0, \alpha)\right)$ such that

$$
|f(t, x)-f(t, y)| \leq \psi(t)|x-y|
$$

For convenience, we introduce the following notation:

$$
\begin{aligned}
& c_{\alpha}=\frac{1}{\Gamma(\alpha)}\left(\frac{1-q_{1}}{\alpha-q_{1}}\right)^{1-q_{1}}\|\mu\|_{L^{\frac{1}{q_{1}}}}, \quad M_{\alpha}=\frac{1}{\Gamma(\alpha)}\left(\frac{1-q_{2}}{\alpha-q_{2}}\right)^{1-q_{2}}\|\psi\|_{L^{\frac{1}{q_{2}}}}, \\
& T_{0}=\frac{c_{\alpha+\gamma}}{1+\eta^{\gamma} E_{\alpha, \gamma+1}\left(-\lambda \eta^{\alpha}\right)}, \\
& T_{i}=T_{i-1}+c_{\alpha}+\left|I_{i}\right|, \quad i=1,2, \ldots, m-1, \\
& T_{m}=\frac{c_{\alpha}+\mathcal{C} \zeta_{\alpha-\delta}}{\left|E_{\alpha}\left(-\lambda\left(1-t_{m}\right)^{\alpha}\right)-\lambda\left(1-t_{m}\right)^{\alpha-\delta} E_{\alpha, \alpha-\delta+1}\left(-\lambda\left(1-t_{m}\right)^{\alpha}\right)\right|} .
\end{aligned}
$$

Clearly, $T_{0}<T_{1}<\cdots<T_{m-1}$.

Theorem 4.1 Assume that (H1) and (H2) are satisfied, then the problem (1.1)-(1.3) has at least a solution $x \in X$ if $M_{\alpha}<1$. 
Proof Define an operator $\mathcal{F}: X \rightarrow X$ by

$$
(\mathcal{F} x)(t)= \begin{cases}\left(P_{0} x\right)(t)+\left(Q_{0}^{\alpha} x\right)(t), & t \in J_{0}, \\ \left(P_{1} x\right)(t)+\left(Q_{1}^{\alpha} x\right)(t), & t \in J_{1}, \\ \cdots & \\ \left(P_{m-1} x\right)(t)+\left(Q_{m-1}^{\alpha} x\right)(t), & t \in J_{m-1}, \\ \left(P_{m} x\right)(t)+\left(Q_{m}^{\alpha} x\right)(t), & t \in J_{m} .\end{cases}
$$

From Lemma 2.9(ii) and Lemma 3.3, we see that $\mathcal{F}: X \rightarrow X$ is clearly well defined.

Similar to (3.2) and (3.3), combining with Lemma 2.9(i) and (2.4), one can get

$$
\begin{aligned}
& \left|\left(Q_{0}^{\alpha+\gamma} x\right)(t)\right| \leq c_{\alpha+\gamma}, \quad\left|\left(Q_{m}^{\alpha-\delta} x\right)(t)\right| \leq \mathcal{C} \zeta_{\alpha-\delta}, \\
& \left|\left(Q_{k}^{\alpha} x\right)(t)\right| \leq c_{\alpha}, \quad k=0,1, \ldots, m .
\end{aligned}
$$

Setting $B_{r}=\left\{x \in X:\|x\|_{1} \leq r\right\}$, where $r \geq \max \left\{T_{m}, T_{m-1}\right\}+c_{\alpha}$, we shall prove $\left(P_{i} x\right)(t)+$ $\left(Q_{i}^{\alpha} y\right)(t) \in B_{r}$ for any $x, y \in B_{r}$ and $t \in J_{i}(i=0,1, \ldots, m)$.

By Lemma 2.9(i) and (4.2), we have

$$
\left|\left(P_{0} x\right)(t)+\left(Q_{0}^{\alpha} y\right)(t)\right| \leq \frac{c_{\alpha+\gamma}}{1+\eta^{\gamma} E_{\alpha, \gamma+1}\left(-\lambda \eta^{\alpha}\right)}+c_{\alpha}=T_{0}+c_{\alpha} \leq r .
$$

For $t \in J_{1}$, one has

$$
\begin{aligned}
\left|\left(P_{1} x\right)(t)+\left(Q_{1}^{\alpha} y\right)(t)\right| & \leq\left|\left(P_{0} x\right)\left(t_{1}\right)+\left(Q_{0}^{\alpha} x\right)\left(t_{1}\right)+I_{1}\right|+\left|\left(Q_{1}^{\alpha} y\right)(t)\right| \\
& \leq T_{0}+c_{\alpha}+\left|I_{1}\right|+c_{\alpha}=T_{1}+c_{\alpha} \leq r .
\end{aligned}
$$

Repeating the above process, for $t \in J_{i}(i=2, \ldots, m-1)$, we find

$$
\left|\left(P_{i} x\right)(t)+\left(Q_{i}^{\alpha} y\right)(t)\right| \leq T_{i}+c_{\alpha} \leq r .
$$

For $t \in J_{m}$, one sees

$$
\left|\left(P_{m} x\right)(t)+\left(Q_{m}^{\alpha} y\right)(t)\right| \leq T_{m}+c_{\alpha} \leq r
$$

Now, we can see that $\left(P_{i} x\right)(t)+\left(Q_{i}^{\alpha} y\right)(t) \in B_{r}$ for any $t \in J_{i}(i=0,1, \ldots, m)$ and $x, y \in B_{r}$.

Similar to (3.1), for $t \in J_{i}, i=0,1, \ldots, m$, one gets

$$
\begin{aligned}
\left|\left(Q_{i}^{\alpha} x\right)(t)-\left(Q_{i}^{\alpha} y\right)(t)\right| & \leq \int_{t_{i}}^{t}(t-s)^{\alpha-1} E_{\alpha, \alpha}\left(-\lambda(t-s)^{\alpha}\right)|f(s, x(s))-f(s, y(s))| d s \\
& \leq \frac{1}{\Gamma(\alpha)} \int_{t_{i}}^{t}(t-s)^{\alpha-1} \psi(s) d s\|x-y\|_{1} \leq M_{\alpha}\|x-y\|_{1} .
\end{aligned}
$$

This implies that $Q_{i}^{\alpha}(i=0,1, \ldots, m)$ is a contraction mapping.

Let $\left\{x_{n}\right\}$ be a sequence such that $x_{n} \rightarrow x$ in $X$, then there exists $\varepsilon>0$ such that $\left\|x_{n}-x\right\|_{1} \leq$ $\varepsilon$ for $n$ sufficiently large. By (H2), we obtain

$$
\left|f\left(t, x_{n}(t)\right)-f(t, x(t))\right| \leq \psi(t) \varepsilon .
$$


Moreover, $f$ satisfies (H1), for almost every $t \in J$, we get $f\left(t, x_{n}(t)\right) \rightarrow f(t, x(t))$ as $n \rightarrow \infty$. It follows from the Lebesgue dominated convergence theorem that

$$
\left\|\left(P_{i} x_{n}\right)-\left(P_{i} x\right)\right\|_{1} \rightarrow 0, \quad \text { as } n \rightarrow \infty
$$

Now we can see that $P_{i}(i=0,1, \ldots, m)$ is continuous.

Moreover, by Lemma 2.9(ii) and (4.2), $\left\{P_{i} x: x \in B_{r}\right\}$ is an equicontinuous and uniformly bounded set. Therefore, $P_{i}$ is a completely continuous operator on $\left.B_{r}\right|_{J_{i}}(i=0,1, \ldots, m)$. Now, it follows from Theorem 2.11 that problem (1.1)-(1.3) has at least a solution $x \in B_{r}$.

\section{Application}

In this section, we give an example to illustrate the usefulness of our main result.

Example 5.1 Consider the following impulsive boundary problem of fractional order:

$$
\left\{\begin{array}{l}
{ }^{c} D_{*}^{\frac{1}{2}} x(t)+5 x(t)=\frac{1}{6 \sqrt[14]{t}} \sin (3+|x(t)|), \quad \text { a.e. } t \in(0,1] \backslash\left\{\frac{1}{4}\right\}, \\
\Delta x\left(\frac{1}{4}\right)=2, \\
x(0)+I_{0^{+}}^{\frac{1}{3}} x\left(\frac{1}{10}\right)=0, \quad x(1)+{ }^{c} D_{\frac{1}{3}}^{\frac{1}{4}}+x(1)=0 .
\end{array}\right.
$$

Corresponding to (1.1)-(1.3), we have $\alpha=\frac{1}{2}, \gamma=\frac{1}{3}, \delta=\frac{1}{4}, \lambda=5, m=2, t_{1}=\frac{1}{4}, t_{2}=\frac{1}{3}, \eta=\frac{1}{10}$, $f(t, x(t))=\frac{1}{6 \sqrt[14]{t}} \sin (3+|x(t)|), I_{1}=2$.

It is easy to see that $|f(t, x(t))| \leq v(t)$ and $|f(t, x(t))-f(t, y(t))| \leq \psi(t)|x(t)-y(t)|$, where $v(t)=\psi(t)=\frac{1}{6 \sqrt[14]{t}} \in L^{\frac{1}{q}}([0,1])\left(q=\frac{1}{7}\right)$ and $\|\psi\|_{L^{7}}=\frac{27}{6}$. By direct computation, we find that

$$
M_{\alpha}=\frac{1}{\Gamma(\alpha)}\left(\frac{1-q}{\alpha-q}\right)^{1-q}\|\psi\|_{L^{\frac{1}{q}}}=\frac{1}{3 \sqrt{\pi}}\left(\frac{6}{5}\right)^{\frac{6}{7}} \approx 0.22<1
$$

Now, due to the fact that all the assumptions of Theorem 4.1 hold, problem (5.1) has at least a solution.

\section{Acknowledgements}

This work was supported partly by the Natural Science Foundation of China $(11561077,11471227)$ and the Reserve Talents of Young and Middle-Aged Academic and Technical Leaders of the Yunnan Province.

\section{Competing interests}

The authors declare that they have no competing interests.

\section{Authors' contributions}

All the authors contributed equally and significantly in writing this paper. All authors read and approved the final manuscript.

\section{Publisher's Note}

Springer Nature remains neutral with regard to jurisdictional claims in published maps and institutional affiliations.

Received: 23 February 2017 Accepted: 19 June 2017 Published online: 30 June 2017

\section{References}

1. Miller, KS, Ross, B: An Introduction to the Fractional Calculus and Fractional Differential Equations. Wiley, New York (1993)

2. Kilbas, AA, Srivastava, HM, Trujillo, JJ: Theory and Applications of Fractional Differential Equations. North-Holland Mathematics Studies, vol. 204. Elsevier, Amsterdam (2006) 
3. Podlubny, I: Fractional Differential Equations. Mathematics in Science and Engineering, vol. 198. Academic Press, San Diego (1999)

4. Sabatier, J, Agrawal, OP, Machado, JAT: Advances in Fractional Calculus: Theoretical Developments and Applications in Physics and Engineering. Springer, Dordrecht (2007)

5. Samko, SG, Kilbas, AA, Marichev, Ol: Fractional Integrals and Derivatives. Theory and Applications. Gordon \& Breach, Yverdon (1993)

6. Barrett, JH: Differential equations of non-integer order. Can. J. Math. 6, 529-541 (1954)

7. Zhou, Y: Basic Theory of Fractional Differential Equations. World Scientific, Singapore (2014)

8. Mophou, GM, N'Guérékata, GM: On some classes of almost automorphic functions and applications to fractional differential equations. Comput. Math. Appl. 59, 1310-1317 (2010)

9. Ahmad, B, Nieto, JJ: Existence of solutions for impulsive anti-periodic boundary value problems of fractional order. Taiwan. J. Math. 15(3), 981-993 (2011)

10. Liu, YJ, Ahmad, B: A study of impulsive multiterm fractional differential equations with single and multiple base points and applications. Sci. World J. 2014, 194346 (2014)

11. Wang, G, Ahmad, B, Zhang, L, Nieto, JJ: Comments on the concept of existence of solution for impulsive fractional differential equations. Commun. Nonlinear Sci. Numer. Simul. 19, 401-403 (2014)

12. Agarwal, RP, Benchohra, M, Hamani, S: A survey on existence results for boundary value problems of nonlinear fractional differential equations and inclusions. Acta Appl. Math. 109, 973-1033 (2010)

13. Wang, JR, Lin, Z: On the impulsive fractional anti-periodic BVP modelling with constant coefficients. J. Appl. Math. Comput. 46, 107-121 (2014)

14. Wang, J, Feckčan, M, Zhou, Y: Presentation of solutions of impulsive fractional Langevin equations and existence results. Eur. Phys. J. Spec. Top. 222(8), 1857-1874 (2013)

\section{Submit your manuscript to a SpringerOpen ${ }^{\circ}$ journal and benefit from:}

- Convenient online submission

- Rigorous peer review

- Open access: articles freely available online

- High visibility within the field

- Retaining the copyright to your article

Submit your next manuscript at $\gg$ springeropen.com 